\title{
New Aspect in Cartilage Growth “The Invasive Interstitial Type”
}

\section{Soha A Soliman*}

Department of Histology, Faculty of Veterinary Medicine, South Valley University, Qena 83523, Egypt

\begin{abstract}
Growth of hyaline cartilage is achieved either by chondrocyte division "interstitial type of growth" or by perichondrial cell differentiation of mesenchymal cells to differentiated chondrocytes which deposit successive circumferential layers of cartilage matrix "appositional growth". The current study was carried out to illustrate a third type of cartilage growth during development of the air breathing dendritic organ of catfish. To perform this study, samples of the air breathing dendritic organ were collected from catfish (Clarias garie-pinus). Paraffin sections of formalin fixed specimens were examined by light microscopy. During development of the air breathing organ of catfish, the nidus of cartilage formation was represented by a condensation of mesenchymal cells. Some cells differentiate to chondrocytes to form multiple cartilage islets surrounded by mesenchymal tissue which was enclosed by perichondrium. The surrounding mesenchymal cells further on differentiate to chondrocytes to complete the developing elastic cartilaginous unit. Additional mesenchymal cells with chondrocyte potential invade the cartilage at multiple sites to grow the cartilaginous mass interstitially. Moreover, interstitial mesenchymal differentiation occurred at the sites of the vacated lacunae which resulted from the death of chondrocytes and then replaces the hyaline. Interstitial mesenchymal invasion occurred in the hyaline cartilage to be replaced by elastic type. The invasive interstitial differentiation of the mesenchymal cells contributed in cartilage growth, renewal, and replacement. Studying the molecular regulation of the invasive differentiation of the mesenchymal cells may be a useful guide in researches to resolve cartilage disorders problems.
\end{abstract}

Keywords: Cartilage; Interstitial growth; Mesenchymal; Invasion; Catfish

\section{Introduction}

Embryonic mesenchymal progenitors are multipotent cells and exhibit the potential to differentiate into multiple cell lineages. Mesenchymal cells destination depends on the molecular environment that enable cells to acquire phenotypic characteristics and perform the tissue functional requirements. Mesenchymal cells can differentiate to chondrogenic or osteogenic cells to form the skeletal elements. During development of cartilaginous elements, the undifferentiated mesenchymal cells transform to chondrocytes. Chondrocytes secrete specific cartilage proteins and form a cartilage template. Cartilage growth depends on the activities of chondrocytes and perichondria stem cells. Interstitial division of chondrocytes commits elongation of the cartilage tissue "interstitial growth of the cartilage". Perichondral cells differentiate to chondrocytes and lay down circumferential layers of cartilage matrix to increase the cartilage diameter "appositional growth" [1]. Embryonic mesenchymal cells invasion to cartilage template and further differentiation has been reported in a previous study [2]. A similar mode of cartilage growth was described in the present investigation. The current study was performed in the elastic cartilage of the air breathing organ in catfish to clarify the difference between growth of mammalian cartilage and the interstitial mesenchymal differentiation during cartilage growth in the air breathing dendritic organ of catfish.

\section{Material and Methods}

The study was carried out on 8 apparent healthy catfish of different ages. Juvenile cat fishes were caught from the River Nile and transported in a glass pool in the lab. Samples of the air breathing organ (suprabranchial organ) were collected from $13-27 \mathrm{~cm}$ measured catfish (Table 1). The obtained samples were fixed in $10 \%$ formalin buffered solution, dehydrated and impregnated and embedded in paraffin wax. Longitudinal sections of 3-5 $\mu \mathrm{m}$ were undertaken and mounted on glass slides. The sections were kept in incubator at $40^{\circ} \mathrm{C}$, stained with H\&E and examined by light microscopy.

\section{Results}

The air respiratory organ of the catfish appeared as arborizing-like structure associated with the gills and located in the suprabranchial air chamber (Figure 1). Development of this organ depends to great extent on formation of the supporting cartilage units. In the arborizing terminals, the primordia of cartilage elements were formed. The first sign of initiation of cartilage elements is condensation of the mesenchymal cells (Figures 2A and 2B). Some mesenchymal cells differentiate to chondrocytes. The primitive suprabranchial cartilage appears as islets of cartilaginous mass embedded in mesenchymal tissue surrounded by perichondrium. Mesenchymal cells later on transformed to chondrocytes (Figures 2C, 2D and 2E) and deposit a cartilage matrix to form a large cartilage mass. Undifferentiated mesenchymal cells originated from the perichondrium, penetrated the interior of the cartilaginous mass and later on transformed to chondrocytes (Figure 2G). Vascular cells originating from the vascular

\begin{tabular}{|c|c|}
\hline $\begin{array}{c}\text { Early stages of developing } \\
\text { catfish (cm) }\end{array}$ & $\begin{array}{c}\text { Advantages stages of developing } \\
\text { catfish } \mathbf{( c m})\end{array}$ \\
\hline 13.4 & 20.9 \\
\hline 13.9 & 24.0 \\
\hline 15.4 & 26.3 \\
\hline 16.2 & 27.2 \\
\hline
\end{tabular}

Table 1: Length of the developing Catfish.

*Corresponding author: Soha A Soliman, Department of Histology, Faculty of Veterinary Medicine, South Valley University, Qena 83523, Egypt, Tel: 2096 5211281; E-mail: Soha.soliman@yahoo.com

Received June 05, 2014; Accepted July 26, 2014; Published August 05, 2014

Citation: Soliman SA (2014) New Aspect in Cartilage Growth "The Invasive Interstitial Type". J Aquac Res Development 5: 253 doi:10.4172/21559546.1000253

Copyright: @ 2014 Soliman SA. This is an open-access article distributed under the terms of the Creative Commons Attribution License, which permits unrestricted use, distribution, and reproduction in any medium, provided the original author and source are credited. 


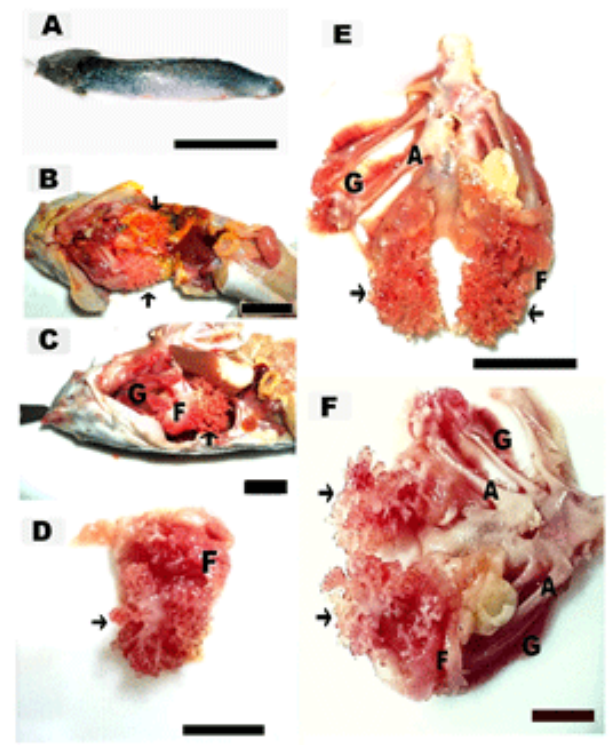

Figure 1: Gross anatomy of the air respiratory organ in catfish.

A: photograph of catfish (Clarias garie-pinus). B: Ventral view of the catfish illustrated the air breathing dendritic organ. C: lateral view of the catfish shows gills and the associated air breathing organ D: shows the air breathing dendritic organ. E,F: respiratory system of catfish. Note: The arrows refer to the air breathing organ. Gill fan $(F)$, Gill filaments $(G)$ and Gill arches (A). Scale bars represent $5 \mathrm{~cm}$ in "A", $2 \mathrm{~cm}$ in "B, C", $1 \mathrm{~cm}$ in "D-E".

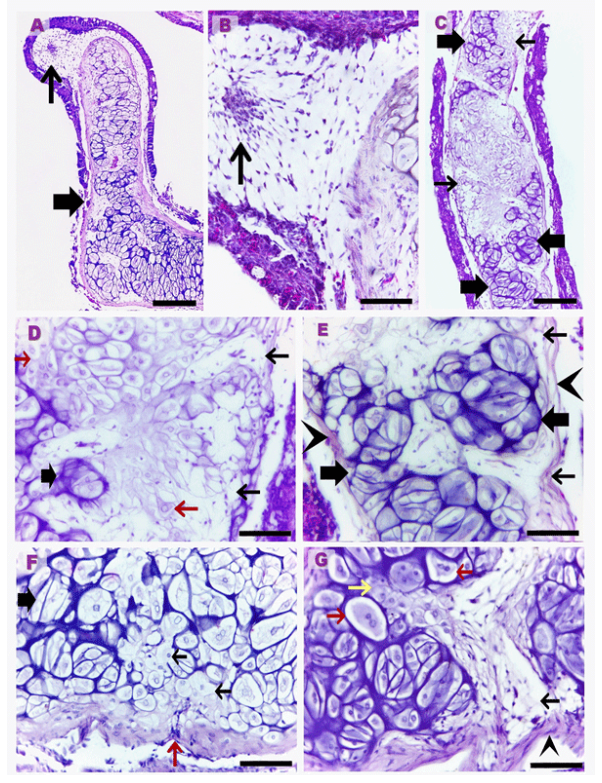

Figure 2: Early differentiation of the cartilaginous support of the air breathing organ.

Paraffin sections of air respiratory organ of catfish measured 13.4 (A), 13.2 $(\mathrm{C}-\mathrm{E}), 14.1(\mathrm{~F}), 14.9(\mathrm{G}) \mathrm{cm}$ in length, stained with H\&E. A, B: The thin arrows refer to the areas of mesenchymal cell condensation. $C$ : The thin arrow point to the differentiating chondrocytes; Note little extracellular cartilage matrix around the differentiating chondrocytes. The thick arrows refer to the cartilaginous masses. D, E, G: The thin arrows refer to areas of the cellular invasion to the growing cartilage. The red arrows refer to differentiating chondrocytes in "D", chondrocytes doublets in "G" and the invading perichondrial mesenchymal cells to the growing cartilage in "F". The yellow arrow refers to the differentiating chondrocytes. The arrow heads refer to the perichondrium. Scale bars represent $80 \mu \mathrm{m}$ in "A, C", $50 \mu \mathrm{m}$ in "B, D-G".

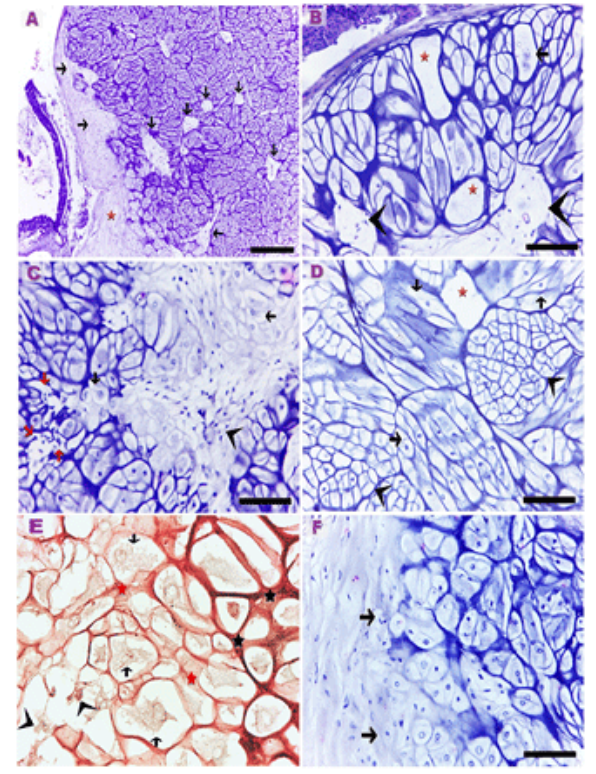

Figure 3: Modes of growth in the cartilage of the air breathing organ. Paraffin sections of air respiratory organ of catfish measured 20.9 (A), 27.3 (B), 14.1 (C), 26.3 (D), 14.9 (G) cm in length, stained with H\&E (A-D,F) and safranin $O(E)$. The thin arrows point to the sites of cellular invasion in "A", terminally differentiated chondrocyte in "B", differentiating chondrocytes in "C, $\mathrm{E}$ ", large groups of chondrocytes in "D" and area of appositional growth in "F". The red arrows refer to the areas of cartilage remodeling. The red asterisks located in the vacated lacunae "B, D" and refer to proteoglycan poor matrix "E". The black asterisks refer to proteoglycan rich matrix "E". The arrow heads refer to the invading mesenchymal cells in "B, C, E". Scale bars represent 80 $\mu \mathrm{m}$ in "A", $50 \mu \mathrm{m}$ in "B-D, F", $20 \mu \mathrm{m}$ in "E".

rich subepithelial connective tissue penetrate the perichondrium and invade the cartilage in association with mesenchymal cells (Figure $2 \mathrm{~F}$ ). The cellular invasion was confined to certain areas in the cartilage and was responsible for the unique enlargement of the cartilaginous mass giving the irregularity of the cartilaginous surface (Figure $3 \mathrm{~A}$ ).

The perichondral cells differentiated to chondrocytes to grow the cartilage appositionally (Figure 3F). Moreover, the invasive undifferentiated mesenchymal cells nearby the perichondrium differentiated to chondrocytes (Figure 3E).

With advancement of age, some hypertrophied chondrocytes reached their terminal differentiation and die leaving vacated lacunae (Figures 3B and 3D). Mesenchymal cells invaded the growing cartilage to occupy the vacated areas and differentiate to chondrocytes (Figure 3B). During cellular invasion, mesenchymal cells can be observed breaking down the septal matrix and opened the lacunae (Figures 5A-5F). Various degrees of differentiation and hypertrophy of chondrocytes and vacated lacunae can be observed in the cartilage (Figure 3D). Chondrocytes doublets were rarely seen (Figure 2E).

In certain locations, cartilage was invaded by vascular cells and the surrounding dense perichondrial tissue to divide it into cartilaginous units (Figures $4 \mathrm{~A}$ and $4 \mathrm{C}$ ) where the covering epithelium invigilated (Figures 4B and 4D).

At the base of air breathing organ, hyaline cartilage was formed and gradually was replaced by elastic type by the mode of invasive interstitial growth. Mesenchymal cells invade the hyaline cartilage and differentiate to chondrocytes capable of synthesizing and secreting elastic fibers (Figures 6A-6E). 

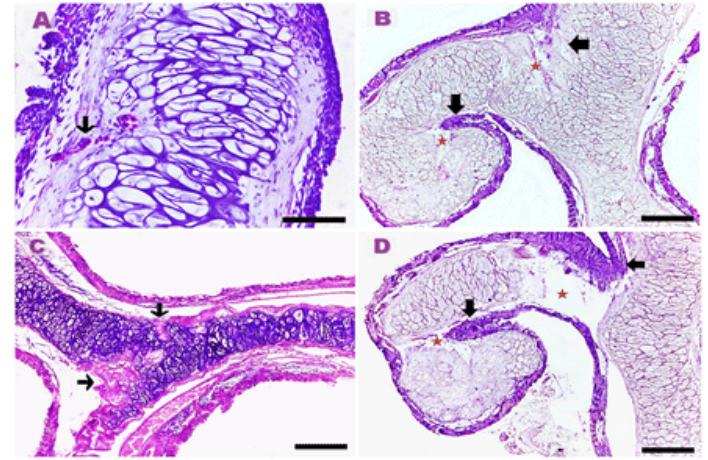

Figure 4: Divisions of cartilaginous units and formation of respiratory compartments in air breathing organ.

Paraffin sections of air respiratory organ of catfish measured $26.3(A), 24.6$ (B D), 20.9 (C) $\mathrm{cm}$ in length, stained with H\&E. The thin arrows refer to areas of vascular and perichondrial CT invasion into the cartilage. The thick arrows refe to epithelial invaginations. The red asterisks refer to divisions of cartilaginous units. Scale bars represent $50 \mu \mathrm{m}$ in "A", $80 \mu \mathrm{m}$ in "B-D".

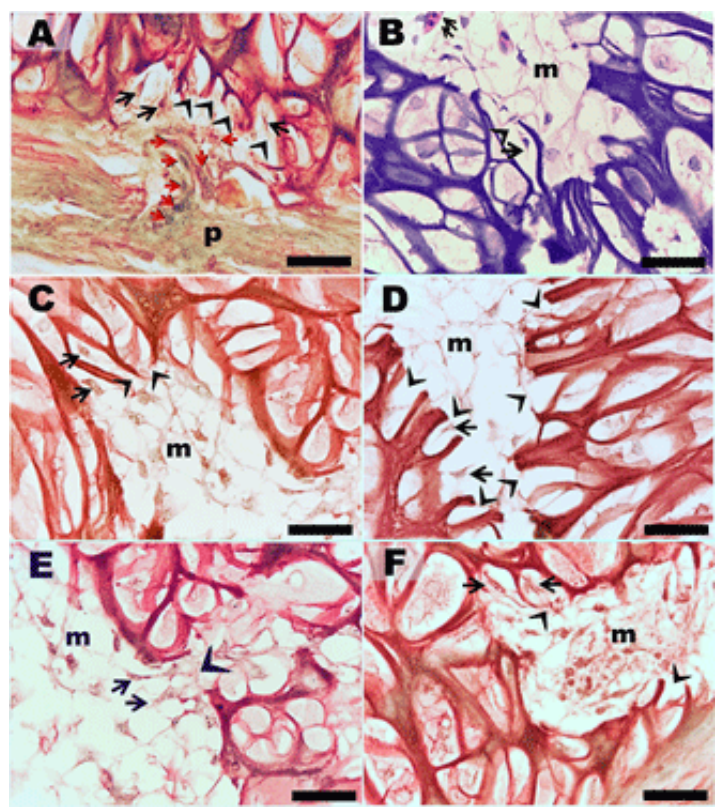

Figure 5: Degradation of cartilage matrix during mesenchymal invasion. Paraffin sections of air respiratory organ of catfish measured 24.6 (A,B), 26.3 $(C, F), 20.9(D, E) \mathrm{cm}$ in length, stained with safranin O "A, C-F" and H\&E "B". The black arrows refer to penetration of mesenchymal cells into lacunae. The red arrows refer to the perichondral originating undifferentiated cells. The arrow heads refer to the opened lacunae. The double arrow refers to vascular inasion. m: mesenchymal cells. Scale bars represent $20 \mu \mathrm{m}$.

\section{Discussion}

Developmental events of skeletal elements in mammals and avian species have been investigated in many previous studies; however Aquarian species had not taken a great interest. In the current investigation, I will focus on the mode of cartilaginous development and growth in the dendritic air breathing organ in catfish and compared with the sequential events and commonly known modes of cartilage growth. In the present study, Mesenchymal cell condensation marked the site of cartilaginous development. Multiple

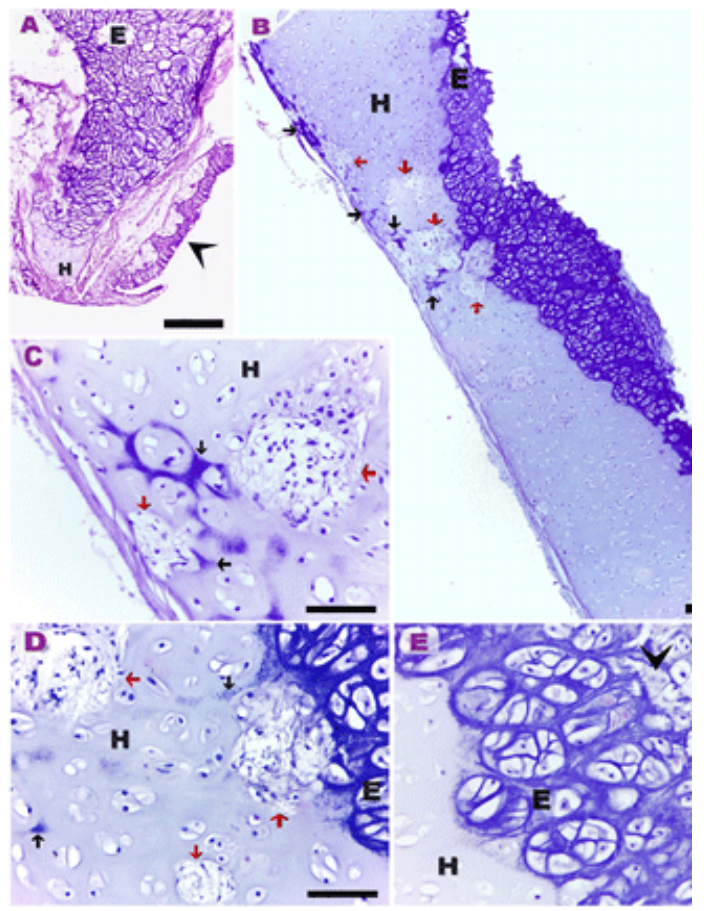

Figure 6: Replacement of hyaline cartilage by elastic type in air breathing organ.

Paraffin sections of air respiratory organ of catfish measured 24.6 (A), 26.3 $(B-E) \mathrm{cm}$ in length, stained with $\mathrm{H} \& \mathrm{E}$. The arrow head refers to the respiratory epithelium in "A" and differentiating chondrocytes of elastic cartilage in "E". The red arrows refer to areas of interstitially located mesenchymal cells. The black arrows refer to the matrix of the newly formed elastic cartilage. Note: "H" hyaline and "E" elastic cartilage. Scale bars represent $80 \mu \mathrm{m}$ in "A", $200 \mu \mathrm{m}$ in "B", $20 \mu \mathrm{m}$ in "C" $50 \mu \mathrm{m}$ in "D".

focal areas of mesenchymal cells differentiate to chondrocytes to form cartilaginous islets interspersed among undifferentiated mesenchyme and surrounded by a delicate perichondrium. Unlike the mammalian and avian species, mesenchymal cell condensation was followed by cartilage primordia which contain pre-chondrocytes at the same stage of differentiation and activity secreting the cartilage matrix and surrounded by perichondrial cells $[1,2]$.

Growth and chondrocytes ordination in cartilage in the air breathing organ was unusual. During cartilaginous growth, multiple sites of cellular invasion can be detected where undifferentiated mesenchymal cells transformed to chondrocytes and secrete cartilage matrix. However, interstitial growth of chondrocytes in the cartilages of the dendritic respiratory organ was rarely observed and isogenous groups or cell nests were not the usual form of chondrocytic organization. Unlike mammals and avian species, interstitial cartilaginous growth of air breathing organ depended primarily on the interstitial invasion and differentiation of the mesenchymal cells. Perichondrial cells contributed in appositional growth in co-ordination with peripheral mesenchymal cells differentiation. Unlike mammals, appositional growth occurs only through the activities of the perichondrial cells $[3,4]$. The invasive interstitial differentiation of mesenchymal cells resulted in a characteristic organization of hypertrophied chondrocytes in the cartilage of the air breathing organ as they were observed in groups of small and large hypertrophic chondrocytes and vacated lacunae. A different organization is usually observed in mammalian cartilages. Appositional growth is responsible for arrangement of the 
immature chondrocytes peripherally and gradually chondrocytes undergo maturation toward the center of the cartilaginous core [3].

Invasive mesenchymal differentiation was recorded in a previous study during growth of cartilage templates of the femur and tibia in quail embryos. Mesenchymal cells of periosteal origin invade the interior of the cartilage template. The cellular invasion was confined to the central hypertrophic zone of the cartilage template and was not observed in the proliferating, resting zones or articular cartilage. Mesenchymal invasion aims to provide the cartilage templates with a population of chondrogenic cells to increase the cartilage diameter of the hypertrophic region. It should be mentioned that mesenchymal osteogenic progenitors usually invade the hypertrophic zone during endochondral ossification [5].

In the largest cartilaginous units of the dendritic air breathing organ, hypertrophic chondrocytes died and empty lacunae were invaded by mesenchymal cells which transformed to chondrocytes. The aim of the cellular invasion may be to regenerate and continue renewal of the cartilaginous tissue. The present study suggests that the mesenchymal cells can degrade the matrix in the invasive pathway. Degeneration of elastic cartilage of the air breathing organ represented by break down of the thin septal matrix, unlike the abundant matrix in hyaline cartilage loss the affinity of staining with proteoglycan reacting stain. The proteolytic activity of the mesenchymal cells has been studied by [6] and is required for division and growth of the tissues [7].

Certain areas of cellular invasion carried the vascular branches from the vascular rich subepithelial connective tissue to the interior of the cartilaginous tissue. The dense perichondrial connective tissue and the associated vascular cells and epithelial covering penetrate the cartilage divide it into masses. This type of invasion may aim to redistribute new vascular elements and the prospective respiratory lamellar epithelial covering to increase the respiratory surface and create new respiratory compartments to adopt the respiratory function.
In the present study, the interstitial differentiation of mesenchyaml cells was observed in the basal hyaline cartilage in the dendritic respiratory organ. Cellular invasion provides the hyaline cartilage with chondrogenic cells which can deposit elastic fibers. The elastic matrix has been detected by Verhoeff's elastic stain [8].

In conclusion, the dynamics of mesnchymal tissue play a central role during development of the cartilaginous support of the dendritic air breathing organ. Invasive interstitial differentiation of the mesenchymal cells was responsible for growth, regeneration and replacement of the cartilage.

\section{References}

1. Singh I (2010) Text book of Human Histology: (with Color Atlas and Practical Guide). Jaypee Brothers Publishers, New Delhi, India.

2. Soliman S (2003) Histomorphological and Morphometrical Studies on Endochondral Ossification in the Japanese Quail. Ph.D Thesis, South Valley University, Egypt.

3. Martini (2007) Anatomy and Physiology. REX Printing Company, Philippines.

4. Singh V (2008) General Anatomy. Elsevier India.

5. Carlevaro MF, Cermelli S, Cancedda R, Cancedda DF (2000) Vascular endothelial growth factor (VEGF) in cartilage neovascularization and chondrocyte differentiation: auto-paracrine role during endochondral bone formation. J Cell Sci 113: 59-69.

6. Ghajar CM, Kachgal S, Kniazeva E, Mori H, Costes SV, et al. (2010) Mesenchymal cells stimulate capillary morphogenesis via distinct proteolytic mechanisms. Exp Cell Res 316: 813-825

7. Shi J, Son MY, Yamada S, Szabova L, Kahan S, et al. (2008) Membranetype MMPs enable extracellular matrix permissiveness and mesenchymal cell proliferation during embryogenesis. Dev Biol 313: 196-209.

8. Ahmed EA, Mohamed K, Ahmed SA, Masoud F (2008) Anatomical, light and scanning electron microscopic studies on the air breathing dendretic organ of the sharp tooth Catfish (Clarias gariepinus). J Vet Anat 1: 29-37. 